\title{
Factores decisivos para a escolha do binómio curso/instituição: o caso do ensino superior agrário português
}

\author{
Isabel Ribeiro \\ Instituto Politécnico de Bragança, Portugal
}

\begin{abstract}
Resumo
Este estudo tem como objectivo identificar os determinantes da escolha educacional e apoia-se em dados primários obtidos através da aplicação de um questionário ao universo dos alunos que se inscreveram, pela primeira vez, no ano 2004, em cursos leccionados em instituições vocacionadas para o ensino superior das ciências agrárias (909 alunos). Através da estimação de modelos logit, foram identificados como determinantes da escolha educacional factores pessoais, particularmente o facto do indivíduo estar ou não bem informado sobre os cursos e as instituições a que se pode candidatar; factores de desempenho e académicos, nomeadamente a nota de acesso, motivos de candidatura ao ensino superior e atitudes perante 0 trabalho e os estudos; factores contextuais, designadamente o facto do indivíduo se encontrar em situação de deslocado, os meios de subsistência, a preparação generalista que o curso proporciona, o prestigio do curso, a existência de maior número de vagas no curso e na instituição, a tradição familiar; e, finalmente, factores sócio-económicos, como o nível de rendimento do agregado familiar e a escolaridade dos progenitores.
\end{abstract}

Palavras-chave

Ensino Superior Português; Ciências Agrárias; Logit; Escolha educacional

\section{Introdução}

Nos tempos mais recentes, um dos temas que tem originado grande número de trabalhos de investigação é, sem dúvida, o Ensino Superior. 
Efectivamente, este sector enfrenta inúmeros problemas, nomeadamente a diminuição drástica do número de alunos; a diminuição do financiamento; a perda substancial de qualidade; consequência, para vários autores, do grande crescimento verificado nos anos 80 e 90 do século passado; entre outros. Por outro lado, King (1995) e Brito (2001) consideram que o ensino superior assumiu centralidade devido aos desafios que se the colocam num meio envolvente altamente dinâmico, nomeadamente os do mundo da globalização e da era do conhecimento que, na opinião de Rodrigues (1998), terá começado a emergir em Portugal a partir dos anos 70 do século XX. São inúmeros os estudos desenvolvidos em diversos países que têm dado conta da existência de uma ligação significativa entre educação e desenvolvimento económico. São vários os documentos da OCDE que mostram que o desenvolvimento dos países está directamente relacionado com o seu nível de educação, investigação e desenvolvimento (I\&D). De acordo com o PNUD (2004), são, precisamente, os países com taxas de alfabetização mais elevadas que usufruem de rendimentos mais elevados e que ocupam as primeiras posições no Índice de Desenvolvimento Humano (IDH) ${ }^{1}$. Também, na opinião de Rego e Caleiro (2003), os países desenvolvidos são, de um modo geral, aqueles que têm um nível de instrução mais elevado ou os que gastam relativamente mais com a educação e com I\&D. Para a UNESCO (1996), o Ensino Superior é, em qualquer sociedade, um dos motores de desenvolvimento económico, pois, para além de depositário e criador de conhecimentos, é também o instrumento de transmissão da experiência cultural científica acumulada pela humanidade. Na mesma linha, Simão, Santos e Costa (2003), consideram que o Ensino Superior desempenha ou deve desempenhar um papel de especial relevo no desafio global da construção da nova sociedade baseada no conhecimento. Estes autores garantem que o poder na sociedade do conhecimento se concentra nas mãos de quem é capaz de dominar a informação, na medida em que esta se constitui como um recurso estratégico para o bom funcionamento dos governos, das empresas e das instituições.

Neste contexto, várias questões se colocam ao indivíduo que decide dar continuidade aos seus estudos após ter terminado o ensino secundário. "Que curso e que instituição satisfaz melhor as minhas necessidades?". Conhecer os factores que estão por detrás de uma decisão tão importante, 
com implicações quer ao nível do desenvolvimento individual e intelectual do indivíduo quer ao nível do desenvolvimento económico e social de um país, é o objectivo deste artigo. Apesar das limitações dos estudos verticais, a análise estática adequa-se ao objectivo referido uma vez que permite a aplicação do questionário ao universo dos alunos que se inscreveram, pela primeira vez, no ano lectivo 2003/2004, no ensino superior agrário português.

Para a realização do estudo, começa por se enquadrar a temática da escolha educacional com vista à construção de um modelo teórico de factores explicativos que podem exercer influência neste tipo de decisão. Com base na revisão da literatura foi desenvolvido e aplicado um questionário ao universo de alunos matriculados, pela primeira vez, no ensino superior agrário português. A informação assim recolhida permitiu estimar modelos logit ${ }^{2}$ de escolha binária que identificam, por um lado, os factores determinantes na escolha do binómio curso/instituição e, por outro, os factores que foram determinantes para a escolha das duas instituições mais procuradas no ano lectivo em que foi aplicado o questionário.

\section{Factores explicativos da escolha educacional}

As complexidades de escolha educacional receberam ampla atenção na literatura das ciências sociais, especialmente na literatura económica com a teoria do capital humano. Quase sempre os modelos desenvolvidos tinham como base o modelo dos custos/benefícios da educação. No entanto, este modelo foi estendido pelas contribuições individuais dos diversos autores no que diz respeito à introdução de novas variáveis com o objectivo de averiguar o seu impacto na escolha educacional. A nível internacional, a principal ênfase colocada pelos vários estudos incide, essencialmente, em factores de natureza social, económica, pessoal, familiar, académica, contextual e de desempenho.

Segundo as opiniões de Psacharoupoulos (1973), Kodde e Ritzen (1987), Gago (1994), Grácio (1997), Verdú (1998), Albert (2000), Jiminéz e Velasquéz (2000), Rego e Sousa (2000) e Balsa, Simões, Nunes, Carmo e Campos (2001), os factores sócio-económicos, nomeadamente o rendimento familiar, a profissão dos progenitores e o nível educacional dos mesmos são características que influenciam a escolha educacional. Estes autores 
verificaram que, durante o processo de acesso ao Ensino Superior, os concorrentes investem noutros factores para além da excelência escolar, isto é, o valor da média de ingresso representa o passaporte que permitirá o acesso a uma determinada instituição de ensino e curso superior. No entanto, existem factores sociais exteriores que podem condicionar o acesso às melhores oportunidades. No que diz respeito à classe social, os autores concluíram que a probabilidade de ingressar no ensino superior continua a ser mais elevada para os indivíduos originários de classes superiores, observando-se a mesma tendência em relação ao nível de instrução dos pais dos candidatos. Ou seja, o acesso ao ensino superior é influenciado pela origem social. A mesma conclusão havia sido também retirada por Rego e Sousa (2000).

Mora (1996), Mora e VillaReal (1996), Diaz (1987), Latiesa (1989), Verdú (1998), Simão et al. (2003), Kodde e Ritzen (1987), Gago (1994), Grácio (1997), Lopes (2001) e Portugal (2004) demonstraram que o mercado de emprego, nomeadamente o estar bem preparado para o exercício de uma profissão, ter a oportunidade de arranjar emprego, ter êxito na carreira profissional, arranjar um emprego bem remunerado, são factores que exercem grande influência na escolha educacional. Valle e Rebelo (2001) defendem que a preocupação com o mercado de emprego está presente nos jovens, não só durante os últimos anos do curso mas também no momento em que estes escolhem a instituição e o curso. Esta escolha é, de acordo com os autores, condicionada pela preocupação dos jovens relativamente à sua inserção futura no mercado de emprego. Por outro lado, Hayes (1997) alega que o estatuto da universidade perspectiva os melhores empregos para quem a frequenta.

A nota de acesso ao ensino superior e a excelência escolar, isto é, o número de reprovações até ao $12^{\circ}$ ano, foram as variáveis incluídas para avaliar a influência dos aspectos relacionados com o desempenho escolar. Efectivamente, segundo Balsa et al. (2001), a média de ingresso no ensino superior representa o passaporte que permite o acesso a uma determinada instituição e a um determinado curso de ensino superior. Relativamente à excelência escolar, trata-se de um indicador da qualidade escolar dos jovens que frequentam o ensino superior público, tal como verificaram Psacharoupoulos (1982), Kodde e Ritzen (1987), Diaz (1987), Gago (1994), 
Grácio (1997), Rego e Sousa (2000), Jiminéz e Velasquéz (2000), Lopes (2001) e Balsa et al. (2001). Neste contexto, à semelhança da média de ingresso ao ensino superior, pretende-se verificar se este indicador é determinante na escolha do binómio curso/escola. Grácio (1997) comprovou que, em média, quanto maior for o aproveitamento maior é a probabilidade de mais investimento escolar. Por outro lado, o aproveitamento e a origem social interagem na medida em que estes influenciam a opção no ensino superior. Ou seja, a probabilidade de enveredar ou não pelo ensino superior dependia tanto mais do aproveitamento anterior quanto mais baixa fosse a origem social.

Para estudar os aspectos de natureza académica e profissional foram incluídas variáveis como a ascensão cultural, o nível de estudos pretendidos pelo inquirido, os motivos de candidatura ao ensino superior e as opiniões do inquirido acerca do trabalho e dos estudos. As duas primeiras tinham sido já testadas por Baía (2000) num estudo que teve como principal objectivo verificar a influência destas e de outras variáveis na escolha de um curso e de uma instituição de ensino superior. As duas últimas haviam sido já testadas pelo ICSUL (1995) e por Diaz (1987), respectivamente. Ambos provaram que estas variáveis exerciam influência na escolha do tipo de ensino, bem como na escolha do curso a frequentar. Por outro lado, relativamente à ascensão cultural, e de acordo com Balsa et al. (2001), tem-se assistido, ao longo destas ultimas quatro ou cinco décadas, a um processo extensivo de mobilidade ascendente em termos de qualificações. Existe uma tendência geral para a elevação do nível de estudos alcançados entre duas gerações precedentes. Também Santos e Baía (2001) argumentam que as expectativas de ascensão social por parte do estudante são um factor determinante na escolha do curso/instituição. Neste contexto, através da variável ascensão em termos culturais, medida pelo nível de instrução dos pais e pelo diploma que o seu descendente ambiciona alcançar, pretende-se saber se esta variável tem influência na escolha educacional.

Kodde e Ritzen (1987), Verdú (1998), Herbert (2000) e Santos e Baía (2001) deram mais um contributo nesta área ao concluírem que os pais exercem influência directa na decisão de escolha educacional dos progenitores. Diaz (1987) e Grácio (1997) demonstraram que as motivações psicológicas e a vocação influenciam, igualmente, a escolha educacional. 
Dias (1997) e Santos e Baía (2001) identificaram alguns factores situacionais passíveis de poderem influenciar a escolha educacional, designadamente a proximidade da residência dos pais, a facilidade de acesso, o número de vagas, a qualidade científica e a possibilidade de poder trabalhar e estudar em simultâneo. Pesquisas mais recentes, levadas a cabo por Balsa et al. (2001), revelam que características como o prestígio, a qualidade e a notoriedade são, normalmente, associadas ao ensino universitário, justificando a escolha de uma instituição universitária em detrimento de uma instituição politécnica.

Na Península Ibérica, Mora (1996), Gago (1994), ICSUL (1995) e Verdú (1998) apontam algumas características pessoais, nomeadamente o género e a idade, como sendo factores com grande influência na escolha educacional. De acordo com Gago (1994: 83), em Portugal,

os rapazes e raparigas com o mesmo aproveitamento escolar e a mesma origem social são, largamente, influenciados nas suas escolhas pelo facto de serem, precisamente, rapazes ou raparigas e que esta influência é maior que a origem social e o aproveitamento juntos.

Relativamente à idade, de acordo com o ICSUL (1995), as situações de ingresso precoce no ensino superior em Portugal verificam-se em maior percentagem em instituições do ensino universitário. Por outro lado, Ribeiro $(2005,2006)$ identificou como determinantes na decisão de escolha do tipo de ensino factores pessoais, como a idade, modos de vida e valores; factores de desempenho e académicos, nomeadamente a nota de acesso, motivos de candidatura ao ensino superior e atitudes perante o trabalho e os estudos; factores contextuais, designadamente o facto do indivíduo viver com os pais, a notoriedade da instituição, a existência de maior número de vagas; e, finalmente, factores sócio-económicos, como o nível de rendimento mensal do agregado familiar.

\section{Metodologia}

A revisão da literatura acerca da escolha educacional permitiu identificar as variáveis independentes a incluir neste estudo que viria a consubstanciar-se num questionário estruturado em quatro partes. A primeira incluía perguntas do foro individual e pessoal; a segunda pretendia fazer o levantamento dos dados sobre a origem geográfica do inquirido e da sua família; a terceira incluía perguntas sobre a origem sócio-económica do 
inquirido, nomeadamente a profissão dos pais, o rendimento familiar mensal e o nível de instrução dos pais; e, por fim, a quarta continha perguntas sobre o desempenho escolar do inquirido e sobre as suas opiniões acerca de matérias como o emprego e o ensino superior. $O$ questionário, com probabilidade de resposta igual para todos os inquiridos, foi administrado directamente ao universo de alunos que se inscreveram, pela primeira vez, no ano lectivo 2003/2004, em instituições de ensino superior, em cursos da área científica das Ciências Agrárias. Obtiveram-se 909 questionários, o que corresponde à totalidade de alunos que se matricularam, pela primeira vez, no ensino superior agrário português nesse mesmo ano lectivo.

Os dados recolhidos foram, posteriormente, tratados recorrendo à estatística multivariada através da estimação de modelos logit com o objectivo de identificar os factores determinantes na escolha educacional e, simultaneamente, perceber a sua capacidade explicativa em termos percentuais. O programa informático utilizado para armazenar, ordenar e tratar os dados foi o SPSS 14.0 (Statistical Package for Social Sciences), pelo facto de ter uma grande capacidade de armazenar dados e possuir uma grande variedade de funções e testes econométricos que permitem estimar modelos, mostrando-se especialmente útil para a realização deste trabalho.

\section{Apresentação e interpretação dos resultados}

Do conjunto de instituições de ensino superior agrário português fazem parte 8 unidades orgânicas do ensino politécnico (Escolas Superiores Agrárias) e 6 unidades orgânicas do ensino universitário (três áreas departamentais, duas faculdades e um instituto). Como pode ver-se na Figura 1, o ensino universitário representa cerca de $27,3 \%$ dos alunos inscritos no ensino superior agrário, pela primeira vez, no ano lectivo de 2003/2004, o que corresponde a 248 alunos, enquanto o ensino politécnico absorve $72,7 \%$, o equivalente a 661 alunos. Na mesma Figura pode ver-se que, no ensino universitário, as três instituições mais representativas, em termos de alunos matriculados, são, por ordem decrescente de importância, a Universidade Técnica de Lisboa - Instituto Superior de Agronomia (UTL — ISA) com 52\%, a Universidade de Trás-os-Montes e Alto Douro - Área de Ciências Agrárias (UTAD) com $21 \%$ e a Universidade de Évora - Área Departamental de Ciências Agrárias (Universidade de Évora) com 19\%. Por fim, a Figura 1 
mostra que, no ensino politécnico, as três instituições mais representativas são a Escola Superior Agrária de Castelo Branco com 20\%, a Escola Superior Agrária de Coimbra com 18,3\% e a Escola Superior Agrária de Beja com $17,5 \%$.
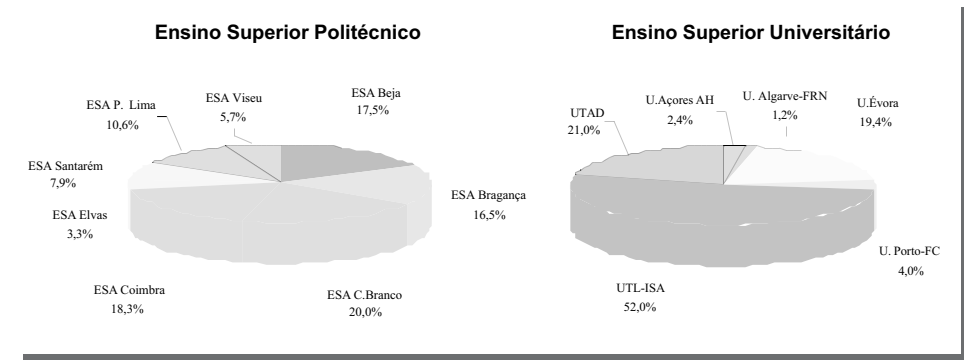

Fonte: Inquérito, 2005

Figura 1 - Distribuição dos alunos do ensino superior agrário por Instituição

No Ensino Superior público, a maioria dos alunos, 54,9\% e 50,9\%, do ensino universitário e do ensino politécnico, respectivamente, ficaram colocados na primeira preferência. Números que contrastam com os verificados pelo ICSUL (1995) no ano lectivo de 1991-1992, uma vez que só o ensino politécnico colocava a maioria dos alunos na primeira preferência, cerca de $67,5 \%$, contrastando com os $35,6 \%$ que ficaram colocados no ensino universitário. Estes resultados ficam a dever-se, sobretudo, ao ajustamento da oferta do ensino superior agrário e ao abrandamento da procura em virtude do decréscimo da população e da diminuição do número de alunos que frequentam o $12^{\circ}$ ano. Enquanto que o ensino universitário tem vindo a ajustar, desde 1996, o número de vagas ao número de candidatos, o ensino politécnico aumentou a oferta de vagas e a diversidade de cursos até 2001, ano a partir do qual o número de vagas tem vindo a diminuir, mas de forma pouco significativa.

O método utilizado para a escolha das variáveis foi o stepwise, processo que, frequentemente, é utilizado em situações em que não são conhecidas as relações ou associações entre as variáveis explicativas e a 
variável explicada. Foi esta, aliás, a razão de ser da recolha de um grande número de variáveis (83 na totalidade, como se pode ver no anexo 1) de onde, posteriormente, seriam analisadas e seleccionadas as mais significativas. Dentro deste método seleccionou-se a variante forward stepwise por partir de um modelo inicial apenas com o termo constante, acrescentando, passo a passo, as variáveis mais significativas até encontrar o "melhor modelo". Este método, segundo Pestana e Gageiro (2005), tem a vantagem de eliminar a hipótese de existência de problemas relacionados com a multicolinearidade 3 . Problemas que, normalmente, põem em causa a significância dos coeficientes estimados. A probabilidade de escolher o ensino universitário é definida através da transformação logit como função dos factores explicativos ${ }^{4}$ :

$$
p=\frac{1}{1+e^{-X \beta}},
$$

onde $Y, X$ e $\beta$ têm os seguintes significados:

$Y$ - Variável binária (assume os valores 0 ou 1).

$X-$ Vector coluna de dimensão $p+1$, em que $p$ é o número de variáveis explicativas e

$\beta$ - Vector paramétrico desconhecido e que se pretende estimar.

Para apreciar a qualidade global do modelo, um dos métodos usuais, de acordo com Johnston e DiNardo (2001), consiste no cálculo da estatística designada razão de verosimilhança $(R V)$, estatística que permite testar a hipótese nula de que os coeficientes são nulos. O cálculo da estatística é o seguinte:

$$
R V=2 L L_{\text {final }}-2 L L_{\text {original }} \sim \chi^{2}{ }_{(K-1)}
$$

em que:

$2 L_{\text {final }}$ - Valor máximo do logaritmo da verosimilhança do modelo estimado.

$2 \mathrm{LL}_{\text {original }}$ - Valor máximo do logaritmo da verosimilhança do modelo que inclui apenas o termo constante.

Convém realçar que o teste à validade global do modelo apenas permite concluir que o seu poder explicativo é maior do que o modelo composto apenas por um termo independente, nada se podendo concluir quanto à significância de cada um dos coeficientes. Para aferir sobre a 
significância individual dos parâmetros testa-se a hipótese nula $H O: \beta_{j}=0$ contra a hipótese alternativa $H 1: \beta_{j} \neq 0$.

Uma vez testada a validade do modelo, quer ao nível de cada parâmetro quer no seu conjunto, deverá, posteriormente, ser testada a qualidade do seu ajustamento. De acordo com Pestana e Gageiro (2005), uma medida comparável ao coeficiente de determinação do modelo de regressão linear $\left(R^{2}\right)$ utilizada é o coeficiente de determinação de Cox \& Snell, que tem como inconveniente o facto de não atingir o valor 1 e que é dado pela expressão seguinte (Pestana e Gageiro, 2005):

$$
R_{\text {Cox ex Snell }}^{2}=1-\left[\frac{2 L L_{\text {original }}}{2 L L_{\text {frinal }}}\right]^{2 / n}
$$

Como forma de ultrapassar esta limitação utiliza-se, frequentemente, o $\mathrm{R}^{2}$ de Nagelkerke que modificou o coeficiente anterior e já atinge o valor 1 . Este coeficiente procura quantificar a proporção de variação explicada no modelo de regressão logística e é calculado através da expressão que se segue (Pestana e Gageiro, 2005):

$$
R_{\text {Nagel ker ke }}^{2}=\frac{R_{\text {cox\& Snell }}^{2}}{1-\left[2 L L_{\text {original }}\right] / n}
$$

Como pode ver-se no Quadro 1, o pseudo $\mathrm{R}^{2}$ de Nagelkerke regista um valor de $18,1 \%$. Por isso, pode afirmar-se que a escolha do binómio curso/estabelecimento é explicado em 18,1\% pelas variáveis independentes. Por outro lado, os valores provam, apesar do baixo valor explicativo do modelo, que a regressão global é altamente significativa em termos estatísticos, uma vez que o Qui-Quadrado (observado) para 9 graus de liberdade é de 80,086 a um nível de significância de 0,0000, sendo superior ao Qui-Quadrado (tabelado ou crítico) para um nível de significância de 5\%, cujo valor é de 16,92.

Os sinais dos parâmetros 5 mostram que o efeito das variáveis: nível de informação, preparação generalista que o curso proporciona e a convicção de que as instituições de ensino superior são locais de aprendizagem e de saber são factores determinantes para a escolha do binómio curso/estabelecimento 
em primeira opção. Por sua vez, factores como a nota de acesso ao ensino superior e a maior probabilidade de entrar no curso motivam os estudantes na escolha do binómio curso/estabelecimento que não conseguem entrar na primeira preferência. Estes resultados são consistentes com os obtidos por Baía (2000) e por Santos e Baía (2001), autores que referem que a facilidade de acesso ao curso e ao estabelecimento determinou a escolha das outras preferências. Por outro lado, Vieira e Brito (2002) e ICSUL (1995) consideram que, frequentemente, os jovens alteram o curso/estabelecimento que indicam como primeira preferência como forma de aumentarem a probabilidade de ingresso. Assim sendo, o facto da nota de acesso ao ensino superior não influenciar positivamente a escolha da primeira preferência não é, de todo, surpreendente.

\section{Quadro 1: Factores determinantes da escolha do binómio Curso/Instituição}

\begin{tabular}{|c|c|}
\hline Variáveis & $\begin{array}{l}\text { Modelo } 1 \\
Y=1 \text {, primeira preferência } \\
Y=0 \text {, outras preferências }\end{array}$ \\
\hline Constante & $-1,392^{* *}$ \\
\hline Nível de informação no acto da candidatura (X12) & $(0,023) \quad 0,741^{*}$ \\
\hline Nota de acesso ao ensino superior (X22) & $(0,165) \quad-0,487^{\star *}$ \\
\hline Maior probabilidade de entrar no curso (X41) & $(0,134) \quad-0,249^{\star *}$ \\
\hline Preparação generalista do curso (X43) & $(0,078)^{\star \star} \quad 0,231^{\star \star}$ \\
\hline Maior probabilidade de entrar na instituição (X52) & $(0,074) \quad-0,158^{* *}$ \\
\hline \multirow{5}{*}{$\begin{array}{l}\text { As instituições de ensino superior são locais de aprendizagem e } \\
\text { de saber (X83) }\end{array}$} & $(0,074) \quad 0,299^{* *}(0,094)$ \\
\hline & ${ }_{-}^{2}=L R=80,086$ \\
\hline & g.l. $=9$ \\
\hline & Nível de significância $=0.0$ \\
\hline & $R^{2}$ Nagelkerke $=0,181$ \\
\hline
\end{tabular}

No sentido de se identificarem os factores que foram determinantes na escolha do estabelecimento de ensino foram ainda estimados modelos para duas unidades orgânicas, uma do ensino universitário (Instituto Superior de 
Agronomia) e a outra do ensino politécnico (Escola Superior Agrária de Castelo Branco), por terem sido as instituições de ensino superior que registaram a maior procura em termos de alunos inscritos, pela primeira vez, no ano lectivo de 2003/2004. Os resultados obtidos após estimação das respectivas regressões são os apresentados nos Quadros 2 e 3.

Foi confirmada, estatisticamente, a qualidade dos modelos, pois as respectivas hipóteses nulas foram rejeitadas ao nível de significância de 5\%, isto é, os modelos estimados que incluem os determinantes para a escolha da instituição foram considerados estatisticamente válidos. Os parâmetros considerados estatisticamente significativos a $5 \%$ são os apresentados nos Quadros 2 e 3, respectivamente.

Quadro 2: Factores determinantes da escolha da UTL-ISA

\begin{tabular}{ll}
\hline Variáveis & \multicolumn{1}{c}{ Modelo 2} \\
& $\mathbf{Y}=\mathbf{1}$, UTL - ISA \\
$\mathbf{Y}=\mathbf{0}$, outras instituições \\
\hline Constante & $-21,64^{\star *}$ \\
Nível de estudos da mãe (X14) & $(1,735)-0,151^{*}$ \\
Nível de rendimento (X18) & $(0,077) 0,481^{\star}$ \\
& $(0,134)$ \\
& $2=\mathrm{LR}=340,946$ \\
& g.l. $=3$ \\
& Nível de significância 0.000 \\
& $\mathrm{R}^{2}$ Nagelkerke $=0,682$ \\
\hline
\end{tabular}

1. Desvio Padrão entre parêntesis

2. * * **, parâmetro significativo a $5 \%$ e $10 \%$, respectivamente. 
Quadro 3: Factores determinantes da escolha da Escola Superior Agrária de Castelo Branco

\begin{tabular}{|c|c|}
\hline \multirow[t]{2}{*}{ Variáveis } & Modelo 3 \\
\hline & $\begin{array}{l}Y=1 \text {, ESA Castelo Branco } \\
Y=0 \text {, outras instituições }\end{array}$ \\
\hline Constante & $-4,776^{\star \star}$ \\
\hline Curso $(X)$ & $(2,043) \quad 0,214^{*}$ \\
\hline Vive com os pais em tempo de aulas (X7) & $(0,047)-1,689^{*}$ \\
\hline Meios de subsistência (X11) & $(0,134) 2,929^{\star \star}$ \\
\hline Arranjar um bom emprego (X25) & $(1,137)-0,934^{*}$ \\
\hline Prestígio do curso (X32) & $(0,167) \quad 1,476^{*}$ \\
\hline Tradição familiar (X42) & $(0,419) \quad 0,689^{*}$ \\
\hline Existência de maior número de vagas na instituição (X53) & $(0,139) \quad 1,078^{*}$ \\
\hline Realização profissional (X70) & $(0,223)-0,764^{*}$ \\
\hline \multirow{5}{*}{$\begin{array}{l}\text { As instituições de ensino superior são locais de aprendizagem e } \\
\text { de saber (X83) }\end{array}$} & $\begin{array}{lll}(0,2034) & -0,857^{\star} \quad(0,222)\end{array}$ \\
\hline & ${ }_{-}^{2}=L R=248,95$ \\
\hline & g.l. $=9$ \\
\hline & Nível de significância 0.000 \\
\hline & $\mathrm{R}^{2}$ Nagelkerke $=0,654$ \\
\hline
\end{tabular}

1. Desvio Padrão entre parêntesis

2. * ${ }^{* *}$, parâmetro significativo a $1 \%$ e $5 \%$, respectivamente.

Os Quadros 2 e 3 mostram que as variáveis explicativas conseguem explicar em $68,2 \%$ e em $65,4 \%$ a escolha do ISA e da ESA Castelo Branco, respectivamente.

A apreciação dos sinais dos parâmetros estimados mostra que factores como o nível de rendimento do agregado familiar é favorável à escolha da UTL-ISA, de destacar que cerca de $82 \%$ dos inquiridos que escolheram o ISA localizam-se nos dois escalões de rendimento mais elevados. Igualmente se apurou que o nível de estudos da mãe favorece a escolha de outras instituições. Estes resultados vêm de alguma forma confirmar o que havia sido apurado por Balsa et al. (2001), autores que afirmam que as instituições de ensino superior mais prestigiadas, geralmente as instituições localizadas nas zonas mais centrais e no litoral do país, tendem a ser escolhidas pelos estudantes oriundos de classes mais favorecidas e que o valor de uma boa média de ingresso é o passaporte para ter acesso a essas instituições. 
Tal como mostra o Quadro 3, no caso da ESA de Castelo Branco, o prestígio do curso, a tradição familiar e o curso leva os alunos a escolher esta instituição, especialmente no caso das licenciaturas em Engenharia Alimentar, Engenharia do Ambiente e Engenharia Zootécnica. Estes resultados assentam sobretudo no facto do inquirido conhecer alguém, que lhe é próximo, que possui um determinado tipo de diploma ou que frequenta um curso específico. Isto é, o conhecimento pessoal não deixa de ser importante na decisão de escolha do curso. Por outro lado, o facto do inquirido depender, em termos económicos, exclusivamente dos pais é também motivo da escolha desta instituição, uma vez que cerca de $88,5 \%$ dos inquiridos têm apenas esta forma de subsistência e destes 55\% encontram-se em situação de deslocado. Os resultados mostram, ainda, que a existência de maior número de vagas leva muitos estudantes a escolher este estabelecimento de ensino superior.

Após ter-se recorrido a técnicas ${ }^{6}$ para melhorar e estimar os modelos logit, os resultados mostram que os modelos são estatisticamente válidos e que, na generalidade, todos os parâmetros apresentam o sinal esperado, isto é, os resultados encontrados estão de acordo com o previsto.

\section{Limitações}

Antes de se tecerem as considerações finais, importa referir dois aspectos que condicionaram este estudo e, como tal, obrigam a interpretar os resultados com alguma cautela. Por um lado, a qualidade estática do estudo força a investigadora a algumas reservas quanto a qualquer tipo de generalizações que, eventualmente, possam ser feitas. Geralmente, os investigadores desta temática evitam estudos longitudinais nos quais os dados sobre a escolha educacional são reunidos e verificados ao longo do tempo. Pela sua natureza, a investigação de Ribeiro (2005) que serve de base a este artigo utiliza, igualmente, a análise vertical com recurso a observações estáticas acerca de características pessoais, familiares, contextuais, sócioeconómicas, de desempenho escolar, académicas e profissionais. Por outro lado, aquando da administração do questionário, as instituições praticavam o sistema da propina única. Por essa razão, a investigadora considerara que, nestas circunstâncias, a variável preço era irrelevante. Actualmente, a situação é diferente, pois as instituições portuguesas de ensino superior começaram a diferenciar os preços. A diferenciação verificada nas propinas 
deste tipo de bens (cursos superiores) pretende, de alguma forma, atenuar a diminuição do financiamento resultante da conjugação de factores como a diminuição da procura e os cortes orçamentais.

\section{Considerações finais}

Este trabalho permitiu identificar factores que justificam 0 comportamento do indivíduo quando este decide dar continuidade aos estudos secundários e opta por um curso na área científica das Ciências Agrárias. Mais, este estudo permitiu verificar que a opção por uma instituição e por um curso é condicionada por factores de natureza diversa, designadamente social, económica, pessoal, familiar, académica, contextual e de desempenho. Efectivamente, o primeiro modelo de regressão estimado permitiu identificar, como factores determinantes na escolha do binómio curso/estabelecimento, em primeira opção: a informação que o inquirido possuía sobre os cursos e as instituições aquando da sua candidatura ao ensino superior; a preparação generalista que o curso proporciona e, ainda, o facto do inquirido ter como convicção que as instituições de ensino superior são locais de aprendizagem e de saber. Por outro lado, foram identificados como determinantes na escolha do Instituto Superior de Agronomia o nível de rendimento do agregado familiar. Finalmente, foram confirmados como factores críticos na decisão de escolha da Escola superior Agrária de Castelo Branco: o curso e o seu prestígio, o facto da instituição colocar à disposição dos candidatos um grande número de vagas nos cursos que oferece e os meios de subsistência que o inquirido tem disponíveis. Para além de explicar a escolha educacional, os modelos obtidos são, igualmente, úteis como ferramenta para gestores e políticos que são responsáveis pela tomada de decisão, pois pode contribuir para a elaboração de critérios mais adequados relativamente ao apoio e financiamento das instituições de ensino superior.

\section{Notas}

1 O IDH é uma medida de três dimensões, uma vez que resulta da média ponderada de três índices: o índice da esperança de vida, o índice da educação e o índice do PIB (Produto Interno Bruto). 
2 Modelo de regressão não linear em que a variável dependente endógena é de natureza qualitativa e binária, isto é, a variável dependente assume apenas os valores 0 e 1 que resultam da codificação de posições que significam somente uma ordenação. O método utilizado para estimar os parâmetros é o da máxima verosimilhança. Para mais informações sobre este modelo deve consultar-se Greene (2000)

3 Quando não se consegue isolar o efeito da variação de $Y$ - variável dependente resultante da variação de cada uma das variáveis $X$ - Variável independente.

4 Para um maior desenvolvimento deste modelo consultar Johnston \& DiNardo (2001).

5 No modelo logit para explicar a probabilidade de $Y=1$, apesar dos parâmetros não darem directamente a magnitude do efeito de cada variável explicativa, os seus sinais, positivo ou negativo, indicam o sentido de variação dessa probabilidade, isto é: $Y i=1$ se $\mathrm{yi}^{\star}>0$ e $Y \mathrm{i}=0$ caso contrário.

6 Foram retiradas as observações com valores de resíduos standartizados $>1,96$, para o nível de confiança 0,05 por se tratarem de outliers (Norusis, 1990). Foram ainda consideradas as correlações entre as variáveis independentes para evitar a questão da multicolinearidade, eliminando-se as variáveis que, por estarem correlacionadas entre si, não reduziam significativamente o logaritmo da função de verosimilhança.

\section{Referências}

ALBERT, Cecilia (2000). Higher Education demand in Spain: the influence of labour market signals and family background. Higher Education, Vol. 40, no 2, pp. 147162.

BAÍA, João (2000). Determinantes na Decisão de Compra: O Papel da Família na Escolha de um Curso Superior. Dissertação de Mestrado. Lisboa: Instituto Superior de Ciências do Trabalho e da Empresa.

BALSA, Casimiro; SIMÕES, José; NUNES, Pedro; CARMO, Renato \& CAMPOS, Ricardo (2001). Perfil dos Estudantes do Ensino Superior. Desigualdades e Diferenciação. Lisboa: Edições Colibri/CEOS.

BRITO, Luís (2001). Perspectivas para o futuro do ensino superior de horticultura em Portugal. Comunicação apresentada no III Encontro de Docentes de Horticultura do Ensino Superior, Escola Superior Agrária de Ponte de Lima, pp. 45-55.

DIAS, J. (1997). Análise Conjunta: Aplicação ao Processo de Decisão de um Curso Superior. Dissertação de Mestrado. Lisboa: Instituto Superior de Ciências do Trabalho e da Empresa.

DIAZ, Miguel (1987). El Acceso a los Estudios Universitarios Análisis y Seguimiento de la Demanda en Asturias. Madrid: Ministerio de Educación y Ciencia.

JOHNSTON, Jack \& DINARDO, John (2001). Econometric Methods. New York: McGraw-Hill Company. 
GAGO, José M. (1994). Prospectiva do Ensino Superior em Portugal. Lisboa: Ministério da Educação, Departamento de Programação e Gestão Financeira.

GRÁCIO, Sérgio (1997). Dinâmicas da Escolarização e das Oportunidades Individuais. Lisboa: EDUCA.

GREENE, William (2000). Econometric Analysis. NJ: Prentice Wall.

HAYES, Louis (1997). Higher Education in Japan. The Social Science Journal, vol. 34, no 3, pp. 297-310.

HERBERT, David (2000). School choice in the local environment: headteachers as gatekeepers on an uneven playing field. School Leadership \& Management, vol. 20, ํㅜ 1, pp. 79-97.

ICSUL. Instituto das Ciências Sociais da Universidade de Lisboa (1995). O Desenvolvimento do Ensino Superior em Portugal: Situação e Problemas de Acesso. Lisboa: Ministério da Educação, Departamento de Programação e Gestão Financeira.

JIMÉNEZ, J. \& VELASQUÉZ, M. (2000). Modelling educational choices. A binominal logit model applied to the demand for Higher Education. Higher Education, vol. 40, no 3 , pp. 293-311.

KODDE, David \& RITZEN, Jozef (1987). Direct and indirect effects of parental education level on the demand for Higher Education. The Journal of Human Resources, vol. 23, no 3, pp.356-371.

KING, Roger (1995). What is Higher Education? Strategic dilemmas for the twenty-first century University. Quality Assurance in Education, vol. 3, no 4, pp. 14-20.

LATIESA, Margarita (1989). Demanda de Educación Superior: evaluaciones y condicionamientos de los estudiantes en la elección de carrera. Revista Española de Investigaciones Sociológicas, vol. 46, pp.101-139.

LOPES, Raúl (2001). Competitividade, Inovação e Território. Lisboa: Celta Editora.

MORA, José-Ginés (1996). The demand for Higher Education in Spain. European Journal of Education, vol. 3, ㄲo 3, pp. 341-355.

MORA, José-Ginés \& VILLAREAL, Enrique (1996). Financing for quality: A new deal in spanish Higher Education. Higher Education Policy, vol. 2, pp.175-188.

NORUSIS, Marija J. (1990). SPSS Base System: User's Guide. Chicago: SPSS.

PESTANA, Maria H. \& GAGEIRO, João N. (2005). Descobrindo a Regressão com a Complementaridade do SPSS. Lisboa: Edições Sílabo.

PNUD. Programa das Nações Unidas para o Desenvolvimento (2004). Relatório do Desenvolvimento Humano 2004: Liberdade Cultural num Mundo Diversificado. Lisboa: Mensagem - Serviços de Recursos Editoriais Lda.

PORTUGAL, Pedro (2004). Mitos e factos sobre o mercado de trabalho português: a Trágica fortuna dos licenciados. Boletim Económico, Banco de Portugal.

PSACHAROPOULOS, George (1973). A note on the demand for enrollment in Higher Education. The Economist, pp. 521-525.

PSACHAROPOULOS, George (1982). An analysis of the determinants of the demand for upper Secondary Education in Portugal. Economics of Education Review, vol. 2, no 3, pp. 233-251. 
REGO, Arménio \& SOUSA, Liliana (2000). Desempenho de estudantes universitários: um contributo empírico. Revista Educação, vol. 9, no 2.

REGO, Conceição \& CALEIRO, António (2003). Impactes das Instituições de Ensino Superior no Território: Estudo do Caso da Universidade de Évora. Documento de Trabalho no 2003/02. Évora: Universidade de Évora, Departamento de Economia.

RIBEIRO, Maria I. (2005). Construção de um Modelo da Procura para o Ensino Superior Agrário em Portugal. Tese de Doutoramento. Vila Real: Universidade de Trásos-Montes e Alto Douro.

RIBEIRO, Maria I. (2006). Determinantes da escolha do tipo de ensino: o ensino superior agrário. Comunicação apresentada no 10ํㅡㄹ Congresso de Economia de Castilla y León, Valladolid, 23 e 24 Novembro.

RODRIGUES, Eduardo (1998). Universidade - espelho e motor da sociedade. In P. Conceição; D. Durão; M. Heitor \& F. Santos (Orgs.), Novas Ideias para a Universidade. Lisboa: IST Press.

SANTOS, Maria \& BAÍA, João (2001). Determinantes na decisão de compra: aplicação a um curso superior. Revista Portuguesa de Gestão, III Série, Ano 16, no 3, pp. 92-100.

SIMÃO, José V.; SANTOS, Sérgio M. Dos \& COSTA, António (2003). Ensino Superior: Uma Visão para a Próxima Década. Lisboa: Gradiva.

UNESCO (1996), Educação: Um Tesouro a Descobrir, Porto: Edições ASA.

VALLE, Patrícia \& REBELO, Efigénio (2001). A inserção dos licenciados em Economia ou Gestão no mercado de trabalho. Revista Portuguesa de Gestão, III Série, Ano 16, № 4, pp. 50-58.

VIEIRA, Carlos \& BRITO, Mónica (2002). Estudo sobre os Ingressados na Universidade de Évora, ano lectivo 2001/2002. Évora: Universidade de Évora.

VERDÚ, Cecilia (1998). La Demanda de Educación Superior en España: 1977-1994. Madrid: Ministerio de Educación y Cultura. 
CRITICAL FACTORS IN THE DECISION MAKING ABOUT INSTITUTION AND COURSE: THE CASE OF AGRICULTURAL HIGHER EDUCATION IN PORTUGAL

\section{Abstract}

To identify factors that are critical in the decision making about institution and course is the main objective of this work and it leans on in primary data obtained through the application of an inquiry to the students' universe that enrolled, for the first time, during 2003/2004, in courses of agricultural sciences (909 Students). Regarding the decision choice about the education, the estimation results identified as determinant factors: personal factors like culture and values and the fact of the individual to be informed about courses and institutions that he can frequent; academic performance factors, namely, access note, reasons to the higher education application, and attitudes about work and study; contextual factors, particularly, the fact that the individual does not live with his parents, the subsistence means, the not specific preparation that the course provides, the prestige of the course, the family tradition, the existence of larger supply, and the major probability of entering in the degree course and in the institution; and, finally, social and economics factors as income level and the level of the progenitors' education.

Keywords

Portuguese Higher Education; Agricultural Sciences; Logit; Educational choice 
FACTEURS CRITIQUES DANS LE CHOIX DE L’ÉTUDIANT POUR LES ÉTUDES SUPÉRIEURES: LE CAS DES ÉTUDES AGRICOLES AU PORTUGAL

\section{Résumé}

L'objectif de ce travail est d'identifier les facteurs critiques dans le choix fait par les étudiants en se qui concerne leurs études supérieures dans le domaine des sciences agricoles. Le travail utilise les données obtenues par l'application d'un questionnaire à l'univers des étudiants qui ont enrôlé, pour la première fois, (en 2003/2004), dans des cours de sciences agricoles (909 étudiants). Concernant la décision au sujet de leur éducation, les résultats de l'estimation ont identifié comme facteurs déterminants: i) les facteurs personnels, par le fait de l'individu être ou pas informé au sujet des cours et institutions qu'il peut fréquenter; ii) la performance académique, à savoir, note d'accès, raisons de candidature aux cours supérieures et attitudes au sujet du travail et de l' étude; iii) les facteurs contextuels, notamment le fait de l'individu (l'élève) être ou pas déplacée, les moyens de subsistance pourvus, et la tradition familiale au sujet de la formation, ainsi que la préparation généraliste apportée par les cours, leur prestige et l'existence d'une grande provision de places (et donc d'une probabilité majeure de réussir l'entrée dans le cours); finalement, iv) les facteurs sociaux et l'économiques, comme le niveau du revenu du ménage et le niveau de formation des parents.

Mots-clé

Études Supérieures Portugaises; Sciences Agricoles; Logit; Choix pédagogique

Recebido em Março, 2007

Aceite para publicação em Fevereiro, 2008

Toda a correspondência relativa a este artigo deve ser enviada para: Isabel Ribeiro, Instituto Politécnico de Bragança, Escola Superior Agrária, Campus de Santa Apolónia, Apartado 1172, 5301 855 Bragança, Portugal. E-Mail: xilote@ipb.pt 


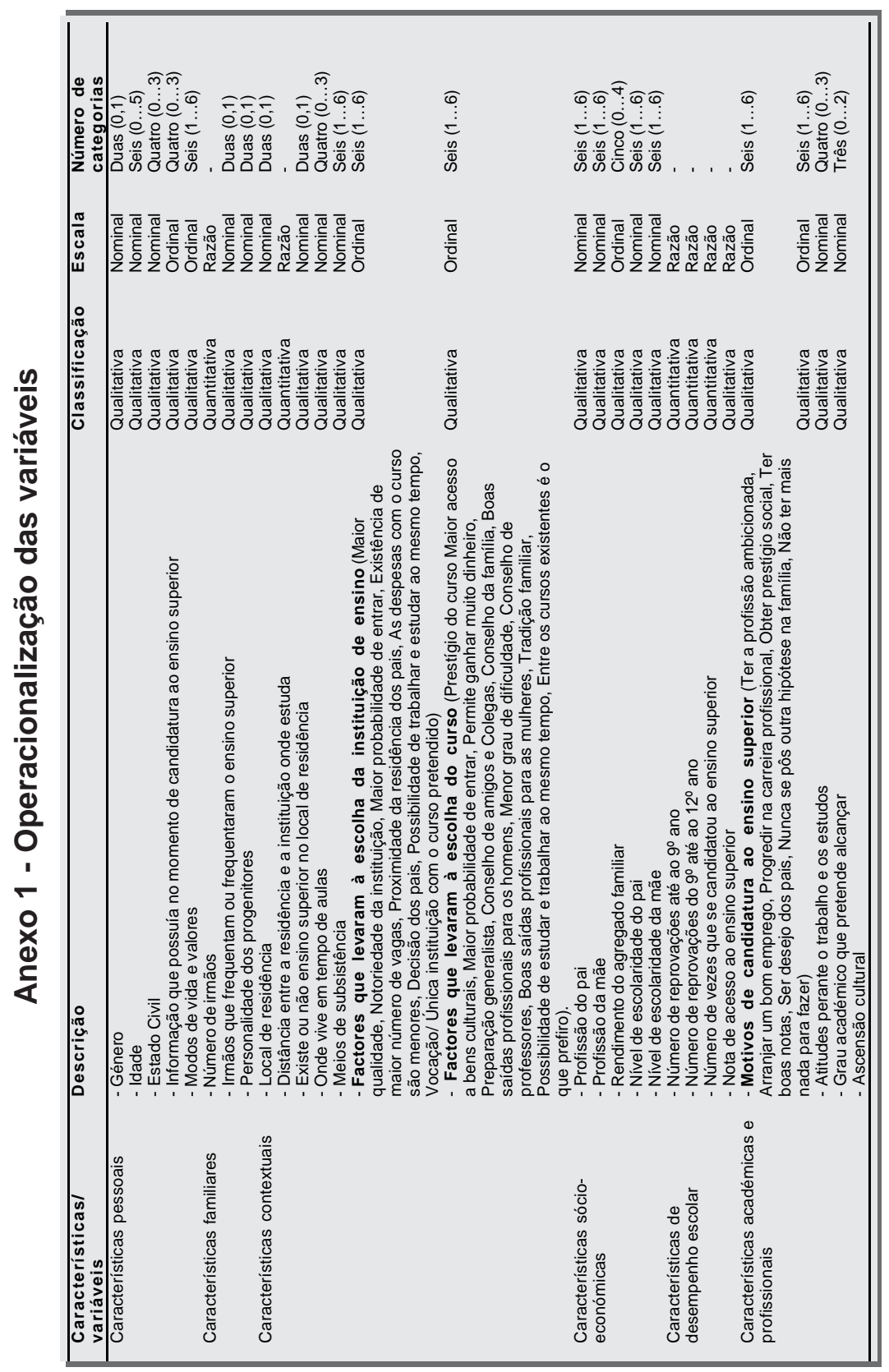

